\title{
Sangrado Digestivo en Niños: Hallazgos Endoscópicos
}

\author{
Gastrointestinal Bleeding in Pediatric: Endoscopy Findings
}

Sarai Elizabeth Umanzor*, Kristell Patricia Hawith**, Ruth Maricela Banegas***

\section{RESUMEN}

Antecedentes: El sangrado del tubo digestivo es una situación alarmante para padres y médicos. Las causas varían de acuerdo a la edad de los pacientes, aunque la mayoría no ponen en peligro la vida, se requiere un enfoque que permita al médico realizar un diagnóstico temprano y valorar la severidad del sangrado, así como la selección de estudios complementarios diagnósticos y terapéuticos. Objetivo: Identificar los hallazgos endoscópicos en niños con sangrado digestivo en el Hospital Nacional Mario Catarino Rivas (HNMCR), período de junio a agosto del 2016. Pacientes y Métodos: estudio descriptivo transversal. La población estudiada fue de 43 pacientes a los que se le realizó estudio endoscópico en el HNMCR en el período de estudio. Resultados: $\mathbf{5 8 \%}$ de quienes se les realizó el estudio endoscópico eran niñas, y $58 \%$ fueron adolescentes. Los hallazgos endoscópicos en los sangrados digestivos altos son gastropatía erosiva $(n=20)$, erosión de esófago $(n=8)$, varices gástricas y esofágicas ( $n=2$ y $n=2$ respectivamente). En las colonoscopias se encontraron pólipos $(n=6)$, y hemorroides externas $(n=1)$. El $66 \%$ de los pacientes estudiados fue el primer episodio de sangrado digestivo. Conclusión: el sangrado digestivo en pediatría tiene causas variadas, desde lesiones tratables en la mucosa del tubo digestivo hasta enfermedades crónicas de las cuales hay que evitar complicaciones a largo plazo.

\section{PALABRA CLAVE}

Sangrado de tubo digestivo alto, tubo digestivo bajo, hallazgos endoscópicos.

\footnotetext{
*Médico residente de Segundo año pediatría

**Médico residente de Primer año pediatría

***Gastroenteróloga Pediatra del Hospital Dr. Mario Catarino Rivas

Dirigir correspondencia: saraumanzor@hotmail.com

Recibido: 17 de diciembre 2016 Aprobado: 25 de febrero 2017
}

\section{ABSTRACT}

Background: Bleeding from the digestive tract is an alarming situation for parents and doctors. The causes vary in accordance with the age of the patients. Usually, gastrointestinal bleedings do not endanger life, but an approach is required that allows the doctor to make an early diagnosis and to evaluate the severity of the bleeding, as well as the selection of complementary diagnostic studies and Therapeutic. Objective: To identify the endoscopic findings in children with digestive bleeding at the Mario Catarino Rivas National Hospital (HNMCR), from June to August, 2016. Patients and Methods: a cross-sectional descriptive study. The study population was the total number of patients $(n=43)$ who underwent endoscopic study in the RMNHR during the study period. Results: $58 \%$ of those who underwent the endoscopic study are girls, 58\% are adolescents. Endoscopic findings in upper gastrointestinal bleeding are erosive gastropathy $(n=20)$, erosion of the esophagus ( $n=8)$, gastric and esophageal varices ( $n=2$ and $n=2$ respectively). In colonoscopies, polyps were found $(n=6)$, and external hemorrhoids $(n=1) .66 \%$ of the patients studied are the first episode of digestive bleeding. Conclusion: gastrointestinal bleeding in pediatrics has a wide range of causes, from lesions in the digestive tract mucosa, treatable and curable, to chronic diseases from which long-term complications need to be avoided.

\section{KEYWORDS}

Upper digestive tract bleeding, low digestive tract, endoscopic findings.

\section{INTRODUCCION}

La hemorragia del sangrado digestivo es motivo importante de consulta en los servicios 
de urgencia pediátrica. ${ }^{(1)}$ La mayoría de las entidades que producen hemorragia digestiva, usualmente no requiere tratamiento quirúrgico, ni conducen a inestabilidad hemodinámica, al no tratarse de hemorragias masivas; pero a pesar de ello, se requiere un enfoque que permita el diagnóstico temprano para la valoración de la severidad y estudios tempranos que permitan detectar la causa y dar tratamiento oportuno. ${ }^{(2)}$

El sangrado digestivo se define como cualquier pérdida de sangre por lesiones que afectan al tubo digestivo que pueden localizarse proximal al ligamento de Treitz considerándose hemorragia digestiva alta (esófago, estómago, duodeno) y la producida distal al ligamento de Treitz como hemorragia digestiva baja, tomando este punto como referencia anatómica, según la localización del sangrado puede manifestarse clínicamente como hematemesis, melena, rectorragia y hematoquezia. ${ }^{(2,3)}$ La etiología puede ser múltiple, la intensidad del sangrado puede ser severa causando inestabilidad hemodinámica (en raras ocasiones) o puede ser leve a moderada sin inestabilidad hemodinámica pero con repercusión importante en el crecimiento y desarrollo del niño, por lo cual es necesario realizar estudios diagnósticos etiológicos y en muchos casos terapéuticos como los que se realizan con procedimiento de endoscopia (alta o baja), siendo factible realizar la toma de biopsia para estudios posteriores. ${ }^{(4)}$

En el HNMCR se realizan endoscopias a diario no contando aun con datos estadísticos del número de endoscopias realizadas, ni hallazgos diagnósticos en los procedimientos realizados, siendo el objetivo de este estudio identificar los hallazgos endoscópicos frecuentes encontrados en niños con sangrado digestivo en el HNMCR en el área de pediatría en el período de junio a agosto del 2016.

\section{PACIENTES Y METODOS}

Se realizó un estudio descriptivo, transversal. La población de estudio fue conformada por niños de ambos sexos menores de 18 años que acudieron al área de Endoscopia del servicio de Gastroenterología Pediátrica del HNMCR, durante el período de junio a agosto del 2016.

El diagnóstico se realizó al presentar cualquier forma de sangrado (hematemesis, hematoquezia, rectorragia y/o melena). Se incluyeron en el estudio todos los pacientes menores de 18 años a quienes se les realizó endoscopia, se excluyeron todos los pacientes a quienes se les realizó endoscopia pero no presentaban signos de sangrado digestivo. El Universo fue conformado por 80 pacientes a quienes se les realizó endoscopia y la muestra fueron 43 pacientes que presentaron clínica de sangrado digestivo (hematemesis, melena, hematoquezia o rectorragia) durante el estudio.

El estudio endoscópico fue realizado por gastroenterólogos pediatras con equipo videoendoscopico en el área de Endoscopia del HNMCR. Previa obtención del consentimiento informado por los padres del sujeto, los datos se recolectaron mediante un instrumento que constaba de 7 preguntas abiertas y 5 de selección múltiple, tomando como variables sexo, edad, signos como hematemesis, hematoquezia, rectorragia, melena, el tipo de estudio (endoscopia alta o baja), hallazgos encontrados al momento de realizar la endoscopia, toma de biopsia y tratamiento indicado.

Los datos obtenidos fueron tabulados con el Software Microsoft Excel 2010, el análisis de datos se realizó con medidas de frecuencia y porcentajes, medidas de tendencia central. Se realizó cruce de variables entre sexo - diagnóstico endoscópico, caso de sangrado digestivo recurrente - hallazgo endoscópico - sexo.

\section{RESULTADOS}

De los 43 pacientes incluidos en el estudio, 25 fueron del sexo femenino y 18 del sexo masculino con un $58 \%$ y $42 \%$ respectivamente, con una proporción hombre: mujer de 1:1.3.

En cuanto a la edad, 58\% fueron adolescentes (12 y 18 años) con media de 16 años, seguido 
por escolares (23\%) entre los 6 y 12 años, preescolares $(11 \%)$ y lactantes $(8 \%)$.

Los signos y síntomas reportados de acuerdo al orden de frecuencia; hematemesis $(66 \%, n=$ $28)$, melena $(16 \%, n=7)$, rectorragia $(16 \%, n=$ $7)$, hematoquezia $(2 \%, n=1)$, lo cual se tomó como indicación para realizar el estudio endoscópico. A 36 (83\%) de los pacientes se les realizó endoscopia alta y a 7 (16\%) endoscopia baja.

Dentro de los hallazgos encontrados en sangrado digestivo alto por orden de frecuencia: gastropatía erosiva $(n=20)$, erosión esófago ( $n: 8)$, varices gástricas $(n=2)$, varices esofágicas $(n=2)$, erosión duodenal $(n=2)$, pólipos gástricos $(n=2)$. En aquellos con sangrado digestivo bajo se encontraron pólipos en intestino $(n=6)$ y hemorroides externas $(n=1)$.

En el género femenino el hallazgo más frecuente fue gastropatía erosiva $(n=15)$, erosión de esófago $(n=8)$. El género masculino el hallazgo más frecuente fue pólipos $(n=6)$ seguido de gastropatía erosiva $(n=5)$ y varices esofágicas $(n=2)$.

El tratamiento médico que recibieron los pacientes fue con inhibidores de bomba (62\% $n=27)$, sucralfato $(23 \% n=10)$. De los 43 pacientes incluidos en el estudio el $20 \%$ ameritó terapia combinada con inhibidores de bomba de protones más sucralfato. y tratamiento contra Helicobacter pylori $(11 \% n=5)$ así como manejo endoscópico quirúrgico con extracción de póli$\operatorname{pos}(18 \% \mathrm{n}=8)$.

El $66 \%$ de los pacientes con sangrado digestivo fueron diagnosticados por primera vez al momento de realizárseles la endoscopia y se les realizó biopsia, el 34\% tenían diagnóstico recurrente. Dentro de los pacientes con diagnóstico recurrente $(n=15)$ el sexo masculino fue el más frecuente $(n=11)$ y los hallazgos endoscópicos más frecuentes fueron pólipos gástri$\cos (n=2)$, poliposis intestinal ( $\mathrm{n}: 6)$, gastropatía erosiva $(n=6)$ y varices esofágicas $(n:=1)$.

\section{DISCUSION}

Existen pocos datos a nivel nacional y local (Hospital Nacional Mario Catarino Rivas) acerca de la frecuencia del sangrado del tubo digestivo en pediatría. A nivel mundial aproximada mente $10 \%$ de los casos se han asociado a una enfermedad sistémica, trastornos hematológicos, septicemias, enfermos graves en la Unidad de Cuidados Intensivos Pediátricos (UCIP); ${ }^{(4)}$ el resto, habitualmente son lesiones de la mucosa: úlceras, erosiones, localizadas en todo el tubo digestivo de las cuales el $10 \%$ son hemorragia digestiva alta, 30\% en intestino delgado, 50\% en región anorrectal. ${ }^{(5)}$ En el área de pediatría del Hospital Mario Catarino Rivas estas causas generan costos hospitalarios elevados ya que aproximadamente se realizan 400 endoscopias al año para diagnóstico o tratamiento en niños.

Las causas de sangrado del tubo digestivo alto anemizante dependen del grupo etario, en los niños mayores de dos años predominan la gastritis medicamentosa o esofagitis debida a enfermedad por reflujo gastroesofágico manifestada clínicamente como epigastralgia o hematemesis como síntoma principal.(6) En este grupo de edad el sangrado del tubo digestivo alto debe hacer sospechar la presencia de várices esofágicas. ${ }^{(5,7)}$ Aunque no hay estudios de su prevalencia, $50 \%$ son de causa extrahepática y se presentan en más del $50 \%$ de los pacientes con cirrosis hepática. ${ }^{(8)}$

En la edad preescolar la presencia de pólipos juveniles como causa de sangrado del tubo digestivo bajo no anemizante, se presenta generalmente con rectorragia intermitente y no se acompaña de dolor. ${ }^{(9)}$ El diagnóstico se establece por medio del tacto rectal, ya que en el $70 \%$ de los casos los pólipos se encuentran en los primeros $20 \mathrm{~cm}$ del rectosigmoides y se confirma con colonoscopia y resección del pólipo. ${ }^{(10)}$

Al analizar la distribución por grupos de edades y sexo en nuestra serie se encontró que el mayor número de pacientes se enmarcaba entre los 12 
a 18 años (58\%), predominando el sexo femenino, siendo la hemorragia digestiva alta por gastropatía erosiva la patología más frecuente, se encontró que 2 de los pacientes presentaban varices esofágicas en el grupo etario prescolar lo cual concuerda con la literatura.

La Endoscopia alta es el método de elección para evaluar el origen del sangrado del tubo gastrointestinal superior; la hematemesis es una indicación para realizar una endoscopia en las primeras 24 horas. ${ }^{(11,12)}$ La hematemesis fue el principal razón de indicación de endoscopia alta durante los meses de junio a agosto del 2016 en el área pediátrica del HNMCR.

En el análisis se observó que la endoscopia alta fue el estudio más frecuente realizado $83 \%$ de los casos.

Bancroft y colaboradores encontraron pacientes con sangrado digestivo de los que $20 \%$ tenía esofagitis, $17 \%$ anomalías de la mucosa gástrica, $6 \%$ úlceras pépticas; $6 \%$ várices esofágicas, $2 \%$ desgarro de Mallory-Weiss y $8 \%$ lesiones no específicas de la mucosa. ${ }^{(13-15)}$ Lo cual es similar a los datos encontrados en nuestro estudio donde se encontró que el mayor porcentaje fue por lesiones en la mucosa gástrica seguido por lesiones en mucosa esófago y un pequeño porcentaje presentaron varices esofágicas.

El sangrado del tubo digestivo alto en pediatría por lo general remite espontáneamente, por lo cual, la endoscopia de urgencia está indicada sólo cuando los resultados pueden influir en una decisión clínica y en realizar intervenciones terapéuticas. ${ }^{(16)}$ Está contraindicada si el paciente se encuentra clínicamente inestable, como en estado de shock, hipovolemia, isquemia miocárdica, o anemia profunda. ${ }^{(17)}$

La rectosigmocolonoscopía permite establecer el diagnóstico en $40 \%$ de los casos, está indicada en casos de hematoquezia y sangrado oculto sin causa demostrada. ${ }^{(19)}$ Las ventajas de endoscopia, alta o baja, son que permite la toma de muestras para biopsias y el tratamiento de algunas lesiones: polipectomía o cauterización de algunas zonas sangrantes. ${ }^{(20)}$

Según lo observado en este estudio a la mayoría de los pacientes se les realizó procedimiento de biopsia para diagnóstico definitivo y $18 \%$ de los pacientes se beneficiaron al recibir tratamiento de polipectomia. De todo ello se deduce la necesidad de asociar técnicas terapéuticas a la endoscopia diagnóstica, y así controlar la hemorragia de forma definitiva.

El tratamiento médico en el sangrado de tubo digestivo alto con lesiones de la mucosa incluye los inhibidores de la secreción de ácido, como bloqueadores $\mathrm{H} 2$, inhibidores de la bomba de protones (IBP). ${ }^{118,21)}$ Se prefiere los IBP sobre los bloqueadores $\mathrm{H} 2$, ya que al inhibir la bomba de protones, se inhiben secundariamente las tres vías de producción de ácido. Se ha descrito que al aumentar el pH gástrico, en especial por encima de 6 , se prolonga el tiempo de formación del coágulo de fibrina, lo cual mejora la homeostasis local y de esta forma se controla el sangrado recomendando su uso en todos los sangrados del tubo digestivo alto, leve, moderado o severo. ${ }^{(22)}$ En nuestro estudio se pudo comprobar que todos recibieron tratamiento médico según las pautas de tratamiento encontradas en la literatura, así como terapia contra Helicobacter pylori siendo causa de sangrado digestivo que amerita tratamiento erradicador, sin duda alguna la estrategia terapéutica que ofrece mejores resultados es la combinación del tratamiento farmacológico con el endoscópico.

En conclusión el sangrado digestivo en pediatría, amerita diagnosticarse oportunamente ya que las etiologías son muy variadas desde lesiones en mucosa en tubo digestivo, tratables, que pueden curarse; hasta enfermedades crónicas de las cuales hay que evitar complicaciones a largo plazo, así como disminuir los factores que pueden intervenir en el adecuado crecimiento y desarrollo del niño. Es de impor- 
tancia dar a conocer en este estudio los signos y hallazgos endoscópicos frecuentes encontrados en niños que acuden al HNMCR, así como la necesidad de perfeccionar los métodos endoscópicos para el diagnóstico y tratamiento de urgencia con el fin de brindar una mejor atención a cualquier paciente con sangrado digesti- vo, (22) profundizando en el conocimiento del tema y sobre todo conocer las características de la población que acude al hospital, disminuyendo morbilidad y mortalidad de esta afección así como las complicaciones a corto y largo plazo.

\section{BIBLIOGRAFÍA}

1. Sánchez $M$, Martín $P$, Ramírez $F$, Prieto $G$, Ruiz M, Pereda A, et al. Protocolos diagnóstico- terapéuticos de gastroenterología, hepatología y nutrición pediátrica de la Sociedad Española de Pediatría. 2a ed. Madrid: Ergon; 2010:

2. Jovel-Banegas LE, Cadena-León JF, Cázares-Méndez JM, Ramírez-Mayans JA, Cervantes-Bustamante R, Zárate-Mondragón FE et al. Sangrado del tubo digestivo en pediatría: diagnóstico y tratamiento. Acta Pediatr Mex. 2013;34(5): 280-288.

3. Kiratli PO, Aksoy T, Bozkurt MF, Orhan D. Detection of ectopic gastric mucosa using 99mTc pertechnetate: review of the literature. Ann Nucl Med. 2009; 23(2):97-105.

4. Mougenot JF, Olshwang S, Peuchmar M. Intestinal tumors: intestinal polyps and polyposis. En Walker: Pediatric gastrointestinal disease: pathology, diagnosis, management. 4th ed. Shelton: USA; 2010. p.214-223

5. Thomson $M$, Fritscher-Ravens $A$, Mylonaki $M$, Swain P, Eltumi $M$, Heuschkel $R$, et al. Wireless capsule endoscopy in children: a study to assess diagnostic yield in small bowel disease in paediatric patients. J Pediatr Gastroenterol Nutr 2007;44(2): 192-7.

6. Velazco-Benitez CA, Guerrero-Lozano R,
Daza-Carreño W. La gastroenterología pediátrica en Colombia. Rev Gastrohnup. 2011;13(3): 173-5.

7. Moreno-Villares J, Polanco I. Paediatric gastroenterology: an atlas of investigation and management. Oxford: Clinical Publishing services; 2009.

8. ASGE Standards of Practice Committee, Fisher L, Lee Krinsky $M$, Anderson MA, Appalaneni V, Banerjee $S$, et al. The role of endoscopy in the management of obscure Gl bleeding. Gastrointest Endosc. 2010; 72(3):471-479.

9. Hwang JH, Fisher DA, Ben-Menachem T, Chandrasekhara V, Chathadi K, Decker GA, et al. The role of endoscopy in the management of acute non variceal upper Gl bleeding. Gastrointest Endosc. 2012;75(6): 1132-8.

10. Silva-García G. Protocolos diagnóstico terapéuticos de gastroenterología, hepatología y nutrición pediátrica de la Sociedad Española de Pediatría. Madrid: Ergón; 2008: 63-77.

11. Espinosa PN, Miquel I. Hemorragia digestiva por várices esofágicas en pediatría. Gastroenterol Latinoam. 2007;18(1):8992.

12. González A, García N, Esteban S. Hemorragia gastrointestinal. En: Asociación Españo- 
la de Pediatría, Sociedad Española de Urgencias Pediátricas. Protocolos diagnóstico-terapéuticos de urgencias de pediatría [Internet]. 2a. ed. España: Ergón;2010 [Consultado el 2 de septiembre del 2016]. p. 103-14. Disponible en: https://www. aeped.es/sites/default/files/documentos/ hemorragia_gastrointestinal.pdf.

13. Bhat $M$, Larocque $M$, Amorim $M$, Herba $K$, Martel M, De Varennes B, et al. Prediction and prevention of upper gastrointestinal bleeding after cardiac surgery: a case control study. Can J Gastroenterol. 2012; 26 (6):340-4.

14. Grimald-Bensouda $L$, Abenhaim L, Michaud L, Mouterde O, Jonville-Béra AP, Giraudeau $B$, et al. Clinical features and risk factors for upper gastrointestinal bleeding in children: a case-crossover study. Eur J Clin Pharmacol. 2010; 66(8):831-7.

15. Yazbeck N, Mahfouz I, Majdalani M, Tawil A, Farra C, Akel S. Intestinal polypoid arteriovenous malformation: unusual presentation in a child and review of the literature. Acta Paediatr. 2011;100(9): 141-4.

16. Restrepo R. Multimodality imaging of vascular anomalies. Pediatr Radiol. 2013; 43(1):S141-54.
17. Itzkowitz S, Potack J. Colonic polyps and polyposis syndromes. En: Sleisenger and Fordtran's gastrointestinal and liver disease. 9th ed. Philadelphia: Elsevier; 2010. p. 2176-87.

18. Amaya Sorto T, García Bacallao E, Lazo del Vallín S, González Fabián L, Torres Fernán$\operatorname{dez} \mathrm{M}$, et al. Familial adenomatous polyposis found in Cuban children. Rev Cubana Pediatr. 2014;86(3):325-335.

19. Lee WS, Zainuddin H, Boey CC, Chai PF. Appropriateness, endoscopic findings and contributive yield of pediatric gastrointestinal endoscopy. World J Gastroenterol. 2013; 9(47):9077-83.

20. Ramirez N, Alparo I. Hemorragia digestiva asociada a Helicobacter pylori en lactantes menores de 6 meses. Rev Bol Ped. 2006;45(1):24-6.

21. Harris PR, Hodgson MI, Soriano H, Gana JC. Gastroenterología y nutrición como una sola especialidad de la pediatría. Rev Chil Pediatr. 2010;81(5): 387-90.

22. Franciosi JP, Fiorino K, Ruchelli E, Shults J, Spergel J, Liacouras CA, et al. Changing indications for upper endoscopy in children during a 20-year period. J Pediatr Gastroenterol Nutr. 2010;51(4):443-7. 